\title{
Postoperative characteristics of combined pharyngoplasty and tonsillectomy versus tonsillectomy in children with obstructive sleep apnea syndrome
}

\author{
Yan Shu, MD ; Hong-Bing Yao, MMa; Da-Zhi Yang, $M M^{a}$ and Bing Wang, $M M^{a}$
}

\begin{abstract}
Purpose. The study aims to identify the postoperative advantages of tonsillectomy in conjunction with pharyngoplasty and tonsillectomy alone in children with obstructive sleep apneas.

Methods. In a prospective observational study, patients who met the study criteria were randomly divided into two groups: tonsillectomy and pharyngoplasty group, and tonsillectomy alone group. In both groups, adenoidectomy was also performed in patients with adenoid hypertrophy. Differences in their healing processes in the pharyngeal wound and their hemorrhage proportions were compared. Furthermore, postoperative velopharyngeal function was also assessed.

Results. Pharyngoplasty together with tonsillectomy was performed in 328 children with obstructive sleep apnea from tonsillar hypertrophy, and tonsillectomy was performed in 275 children. These cohorts did not reveal any significant demographic differences between groups. Furthermore, blood loss was found to be significantly decreased in the tonsillectomy and pharyngoplasty group $(P<0.01)$, and the healing process was markedly shorter. Postoperative velopharyngeal function was not affected.

Conclusions. Tonsillectomy in conjunction with pharyngoplasty reduced the duration of the healing process and blood loss, compared with tonsillectomy alone; however, postoperative complications did not increase. We consider pharyngoplastyin conjunction with tonsillectomy has great potential in the treatment of children with obstructive sleep apnea.
\end{abstract}

Key words: Pharyngoplasty, tonsillectomy, child, obstructive sleep apnea.

http:/ / dx.doi.org/10.5546/ aap.2018.eng.316

E-mail address:

Bing Wang:

bingwangdoc@163.com

Funding:

This study was

supported by Medical

Project of Chongqing

Municipal Health

Bureau, China

(NO: 20120116).

Conflict of interest:

None.

Received: 7-22-2017

Accepted: 3-19-2018 age when tonsil hypertrophy and adenoids are more commonly observed. ${ }^{2}$ If left untreated, OSA can lead to serious morbidity and affect the cognitive, neurobehavioral and cardiovascular systems. ${ }^{3,4}$ OSA in children is primarily attributed to adenotonsillar hypertrophy, ${ }^{5}$ and as such, tonsillectomy with or without adenoidectomy (T\&A) is currently the most common treatment for children with OSA. ${ }^{6}$ The standard procedure for adenotonsillectomy includes the removal of the palatal tonsils and adenoids.

Although tonsillectomy is one of the most commonly performed otolaryngologic procedures, some complications may arise including postoperative bleeding, pain, decreased oral intake, postobstructive respiratory compromise, velopharyngeal insufficiency and psychological trauma. ${ }^{7,8}$ In order to reduce these complications, various techniques have been continuously evolved, including coblation tonsillectomy, ${ }^{9}$ bipolar electrodissection tonsillectomy ${ }^{10}$ and laser tonsillectomy. ${ }^{11}$ However, no strong evidence supports the use of pharyngoplasty in children with OSA. The term pharyngoplasty refers to closing the tonsillar wound by suturing the anterior and posterior pillars after tonsillectomy. For a long time, the necessity and risk of suturing the tonsillar wound after tonsillectomy has remained controversial.

The aim of this study was to compare the outcomes of tonsillectomy in conjunction with pharyngoplasty with the outcomes of tonsillectomy alone in children with OSA. 


\section{MATERIALS AND METHODS Population}

The population in our study were pediatric patients evaluated and scheduled to undergo tonsillectomy with or without adenoidectomy for OSA in the Children's Hospital of Chongqing Medical University between September 2008 and June 2010. We excluded patients only received adenoidectomy alone and observed the pure tonsil excision or tonsillectomy with adenoidectomy. The indications for all patients included the presence of OSA with tonsillar and / or adenoid hypertrophy. The diagnosis of OSA was based on the history provided by the caregiver (snoring, mouth-breathing, witnessed apneas, enuresis, daytime hypersomnolence, or restless sleep), physical examination, and polysomnographical data analysis results. We chose the patients with obstructive apneahypopnea index $(\mathrm{AHI})>5$ as documented by polysomnography, as well as with all tonsil grades (I-IV). ${ }^{12}$ Exclusion criteria were significant comorbidities or a significant history of recurrent/chronic tonsillitis, in addition to a history of a bleeding disorder. We also ruled out chronic nasal obstruction and other causes of airway obstruction. This study was approved by the local regional ethical committee. Signed informed consent was obtained from parents / guardians of the children. According to the random number table, the children were divided into two treatment groups: pharyngoplasty and tonsillectomy group, and tonsillectomy alone group.

The children were submitted to overnight PSG in the sleep disorders laboratory. The polysomnogram was analyzed using standard techniques. The obstructive AHI was defined as the number of apneas and hypopneas per hour of total sleep time. Adenoid size was determined by a lateral X-ray of neck. The adenoid to nasopharynx ratio $(\mathrm{A} / \mathrm{N})$ is defined as the ratio between maximal adenoid thickness and distance along a line from the posterior-superior edge of the hard palate to the spheno-occipital synchondrosis on the base of the skull. ${ }^{13}$ In our study, patients with $\mathrm{A} / \mathrm{N}>0.65$ were subjected to adenoidectomy.

The data collected included the patient's age, gender, preoperative diagnosis, polysomnography (PSG) data, ranking of speech intelligibility and velopharyngeal insufficiency, and any postoperative complications.

\section{Surgical techniques}

Children from both groups were under general anesthesia and orotracheal intubation, and were placed in the standard supine position during operations. The mouth was opened by a BoyleDavis mouth gag. The soft palate was retracted using two pediatric 9-mm catheters.

In the pharyngoplasty and tonsillectomy group, after the pericapsular dissection plane was identified, pericapsular dissection was performed with outmost care from the superior to the inferior poles of the tonsils. Then, the tonsils were dissected towards the lower pole with a blunt dissector. The tonsils were removed from the tonsillar fossa using a wire snare and then the fossa was packed with a swab dipped in normal saline to achieve hemostasis. Of course, absorbable ties and bipolar forceps were used to secure the hemostasis, when necessary. The anterior and posterior pillars and the organization of the fossa were sutured with a single 4-0 chromic gut suture to secure the hemostasis and close the tonsillar fossa. The suture was not removed after the operation.

In the tonsillectomy alone group, the surgical removal of the tonsil tissue itself and the primary procedure of hemostasis were performed, which is similar to the procedure previously described. Then, further hemostasis of the tonsillar fossa was secured by point hemostasis with bipolar forceps. After complete hemostasis of both tonsillar fossa was achieved, the adenoid was removed through endoscopic surgery.

\section{Assessment of velopharyngeal function Assessment of speech}

We performed speech evaluation and flexible nasopharyngoscopy after obtained the consent of the parents on the fifth day after operation. All of them agree to the exam. Speech assessments were obtained based on routine speech analysis by pediatric speech pathologists, using a scale developed at this institution prior to the acceptance of universal speech parameters. The results of the analysis were graded using a fivepoint scale (0-4), in which 0 indicates normal and 4 indicates severe. A lower score on this scale indicated less dysfunction. ${ }^{14}$

\section{Flexible nasopharyngoscopy}

Both groups underwent pre- and postoperative nasal endoscopy administered by the same pediatric otolaryngologist. Visualization of the velopharyngeal port was performed 
using a fiberoptic flexible nasopharyngoscope. Palatal motion, lateral pharyngeal wall motion and velopharyngeal closure were observed. Velopharyngeal gap size was measured during the maximal closure on phonation. Results were graded using the Golding-Kushner scale. ${ }^{15}$ Gap size categories were defined as large (velopharyngeal closure $<50 \%$ ), moderate (velopharyngeal closure between $50 \%$ and $80 \%$ ), or small (velopharyngeal closure $>80 \%$ ).

\section{Statistical analysis}

Statistical analysis was performed using the statistics program SPSS for Windows. Demographic variables of age and gender were compared by chi-square test. Comparisons of preoperative and postoperative complications such as hypernasality and nasopharyngeal regurgitation were achieved using Fisher's exact test. A $P$-value $<0.05$ was considered statistically significant.

\section{RESULTS}

\section{Cohort demographics}

The demographics of patients included in this study are indicated in Table 1. A total of 682 pediatric patients were evaluated for OSA; 79 patients were excluded because their parents rejected postoperative nasal endoscopy. Hence, the remaining 603 patients were enrolled into this study. Among these 603 patients, 328 patients
(138 females, 190 males) who underwent pharyngoplasty and tonsillectomy were included as the study group, while 275 patients (108 females, 167 males) who underwent pure tonsillectomy with or without adenoidectomy were included as the control group (Figure 1). The mean age of the study and control group was $4.9 \pm 2.1$ years (range $3-11$ years) and $5.13 \pm 2.4$ years (range 3-12years), respectively.

Differences in any of these demographic variables between these two groups were not statistically significant (Table 1).

TABLE 1. Demographic information

\begin{tabular}{|c|c|c|c|}
\hline & Pharyngoplasty & $\begin{array}{c}\text { Pure } \\
\text { tonsillectomy }\end{array}$ & $P$-value \\
\hline No. of patients & 328 & 275 & \\
\hline Age (year) & $4.9 \pm 2.1(3-11)$ & $5.13 \pm 2.4(3-12)$ & NS \\
\hline $\begin{array}{l}\text { Gender, } \\
\text { female/male }\end{array}$ & $138 / 190$ & $108 / 167$ & NS \\
\hline BMI (z score) & $0.72 \pm 1.13$ & $0.75 \pm 1.16$ & NS \\
\hline $\mathrm{A} / \mathrm{N}$ ratio & $0.71 \pm 0.05$ & $0.69 \pm 0.03$ & NS \\
\hline Tonsillar Size & $2.22 \pm 0.13$ & $2.25 \pm 0.15$ & NS \\
\hline $\begin{array}{l}\text { Obstructive AHI } \\
(/ \mathrm{h}, \mathrm{TST})\end{array}$ & $16.2 \pm 4.1$ & $15.95 \pm 4.7$ & NS \\
\hline
\end{tabular}

A/ $\mathrm{N}$ ratio, adenoidal/nasopharyngeal ratio;

NS, not significant; AHI, apnea-hypopnea index;

$/ h$, TST, per hour of total sleep time.

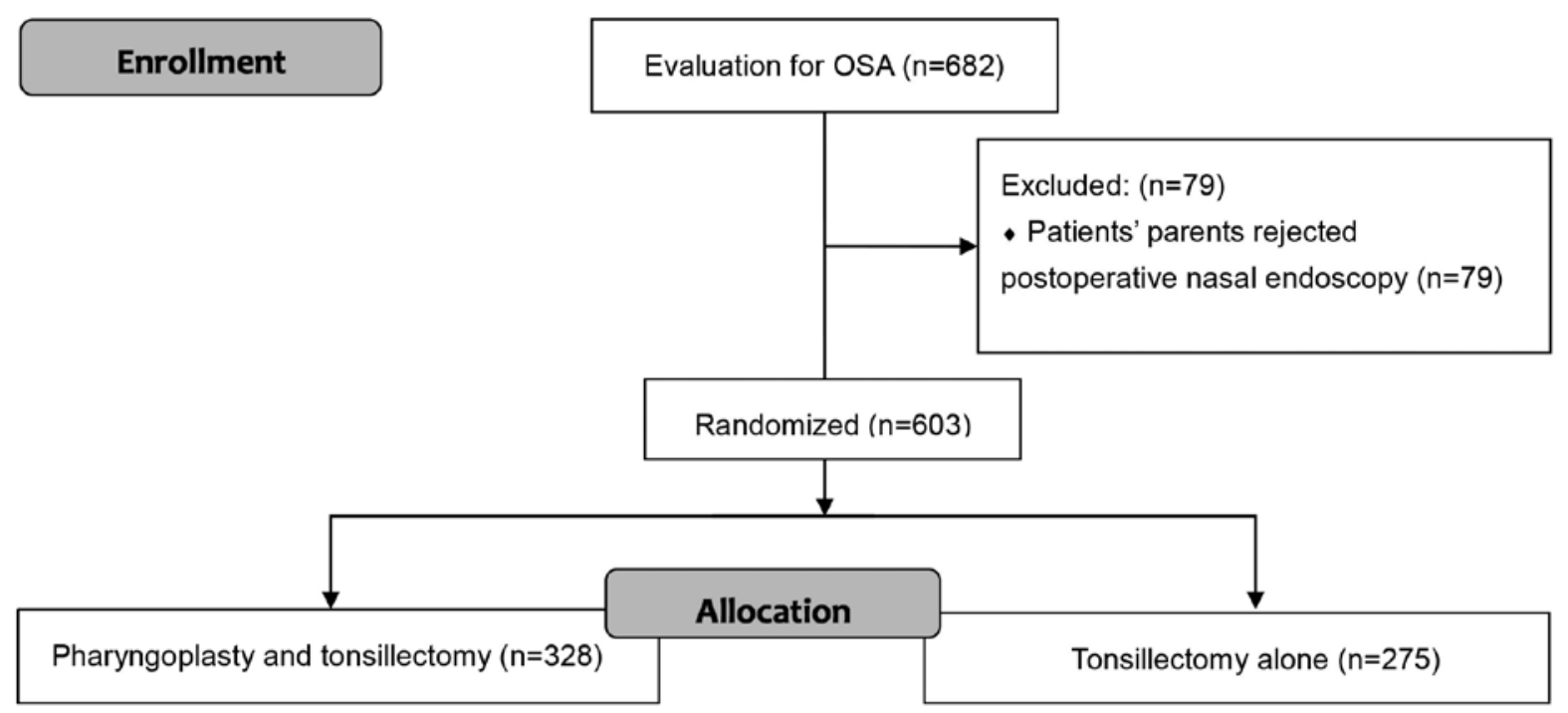




\section{Postoperative hemorrhage}

In primary hemorrhage, the obtained results were statistically significant. The rate for postoperation hemorrhage was $0.91 \%$ (3 patients) in the study group and $4.36 \%$ (12 patients) in the control group. The comparison of these proportions revealed that the difference between these two groups was statistically significant $(P=0.008$, Table 2). Furthermore, delayed hemorrhage occurred in two cases in the control group.

\section{Pharyngeal wound characteristics}

Upon examining the throat at five days postoperation, a large difference in the healing of the pharyngeal wound was found. The wounds in patients who underwent pharyngoplasty and tonsillectomy were clean and almost healed. However, considerable slough or white pseudomembranes were present in the tonsillar fossa in the pure tonsillectomy group. This condition had an impact on post-tonsillectomy hemorrhaging and the amount of time patients spent in the recovery phase of this operation.

TABLE 2. Postoperative hemorrhage (\%)

\begin{tabular}{lccc}
\hline & $\begin{array}{c}\text { Study group } \\
(\mathbf{n}=\mathbf{3 2 8})\end{array}$ & $\begin{array}{c}\text { Control group } \\
(\mathbf{n}=\mathbf{2 7 5})\end{array}$ & \\
\hline Primary hemorrhage & $3(0.91)$ & $10(3.63)$ & 0.025 \\
Delayed hemorrhage & $0(0)$ & $2(0.73)$ & 0.208 \\
All hemorrhage & $3(0.91)$ & $12(4.36)$ & 0.008 \\
\hline
\end{tabular}

\section{Postoperative velopharyngeal function}

With respect to postoperative speech intelligibility, five patients in the study group and three patients in the control group significantly worsened, although no significant trends towards worsening were noted when the outcomes of the two groups were compared $(P=0.733)$. All patients underwent flexible electronic nasopharyngoscopy. Obvious changes in palatal closure, lateral wall movement and velopharyngeal gap sizes were not present between each patient's pre- and posttonsillectomy data (Table 3 and Figure 2). Thus, the difference between these two groups was not statistically significant.

Postoperative nasopharyngeal backflow and hypernasality were not observed in this population.

\section{DISCUSSION}

Although the study of non-operative therapies has become an increasingly viable option, surgery remains the main treatment

TABLE 3. Postoperative change about velopharyngeal function (\%)

\begin{tabular}{lccc}
\hline & $\begin{array}{c}\text { Study group } \\
(\mathbf{n = 3 2 8})\end{array}$ & $\begin{array}{c}\text { Control group } \\
(\mathbf{n}=\mathbf{2 7 5})\end{array}$ & P value \\
\hline $\begin{array}{l}\text { Speech intelligibility } \\
\text { worsened }\end{array}$ & $5(1.52)$ & $3(1.09)$ & 0.733 \\
$\begin{array}{l}\text { Velopharyngeal } \\
\text { insufficiency }\end{array}$ & $0(0)$ & $0(0)$ & \\
$\begin{array}{l}\text { Nasopharyngeal } \\
\text { backflow }\end{array}$ & $0(0)$ & $0(0)$ & \\
$\begin{array}{l}\text { Hypernasality } \\
\text { Hyon }\end{array}$ & $4(1.22)$ & $2(0.73)$ & 0.694 \\
\hline
\end{tabular}

\section{FIGURE 2. Nasopharyngoscopic views during articulation}

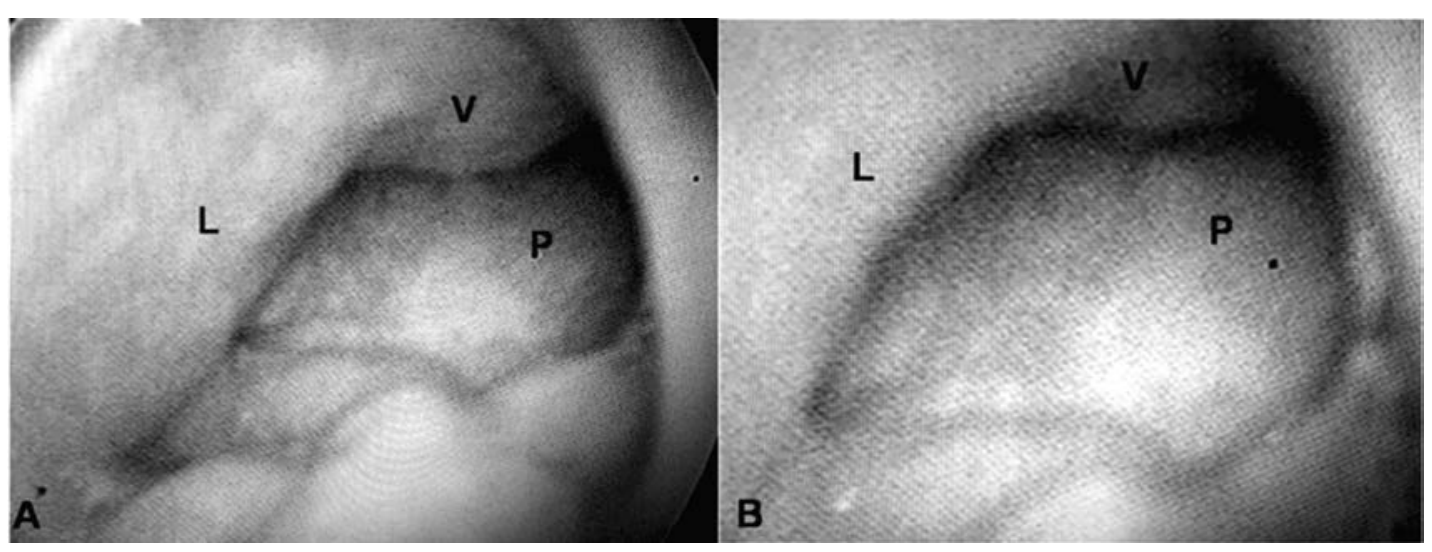

(A) Preoperative view with obliteration of the gap.

(B) Postoperative view with obliteration of the gap (velopharyngeal closure). L=lateral nasopharynx wall;

$\mathrm{P}=$ posterior pharyngeal wall; $\mathrm{V}=$ velum. 
method recommended for children with OSA at present. The discussion of avoiding postoperative complications and surgeryassociated discomforts remains a substantial focal point. Our results provided further support to the performance of combined pharyngoplasty and tonsillectomy for treatment of children with obstructive sleep apnea. Although this procedure is not accepted by some otolaryngologists due to its increased operating time and uncertain advantages, our study demonstrates the benefits of pharyngoplasty combined with tonsillectomy in the prevention of postoperative bleeding and wound healing.

Postoperative hemorrhage is one of the main post-tonsillectomy morbidities including primary or reactionary hemorrhages ( $<24$ hours), and postoperative infections associated with secondary or delayed hemorrhages ( $>24$ hours). ${ }^{16}$

Primary hemorrhage is more brisk and profuse than secondary hemorrhage. Moreover, it occurs when the patient's protective airway reflexes are blunted by post-anesthetic or narcotic effects. ${ }^{17}$ Our results have shown that reactionary hemorrhage was more common than delayed hemorrhage in both populations. Furthermore, delayed hemorrhage appeared primarily in the control group. The rates of hemorrhage were low in the study group (below 1\%). These results may be related to the closing of the tonsillar wound by suturing the posterior and anterior tonsillar pillars, and thereby avoiding any reopening of intraoperative damaged blood vessels. Additionally, Lehnerd $\mathrm{t}^{18}$ considered several instances of dietary trauma related to hemorrhaging. The procedure we chose may also effectively reduce postoperative dietary stimulation.

From the first tonsillectomy described by Celsus in $50 \mathrm{AD},{ }^{19}$ otolaryngologists have become accustomed to not suturing the wounds. Currently, it remains uncertain whether this procedure is really and truly beneficial to wound healing. Tonsillectomy produces an open wound that is open to influence from various factors in a grossly contaminated field. Wound healing relies on the ingrowth of the mucosa from the periphery, which may continue for 1 to 2 weeks after surgery. During the process, the postoperative fossa would undergo a series of complex pathophysiological changes including fibrin clot formation, proliferation, and separation from the underlying granulation tissue. ${ }^{20}$

Patients would also experience varying levels of discomfort. All of these factors may increase the time to complete healing. Although most of the open wounds can be healed, it may be possible to shorten the healing time and level of discomfort. In our study, almost all patients in the pharyngoplasty and tonsillectomy group had completely healed pharyngeal wounds by the fifth day after surgery, while most patients in the control group had tonsillar fossae that remained in the active processing of fibrin clots.

The practice of suturing the tonsillar pillars following tonsillectomy has gradually been adopted in children with OSA by some otolaryngologists. However, whether some adverse effects would arise from this approach including negative effects on postoperative velopharyngeal function is continuously being widely questioned.

Function is usually decided by structure. Structural modification in the upper airway may influence function and physiology to a certain extent after reconstructive surgeries, including uvulopalatopharyngoplasty and pharyngoplasty. Although velopharyngeal insufficiency is an uncommon complication of traditional uvulopalatopharyngoplasty, it is a risk factor for patients when a resection is performed or in patients who have impaired mobility of the palate and/or lateral pharyngeal walls. ${ }^{21}$ One study has shown a lower risk of deterioration in velopharyngeal function among patients without cleft palates after pharyngoplasty tonsillectomies.2. In our study, we tried to assess the velopharyngeal function in children with OSA after pharyngoplasty. However, no statistical significance compared with the control group. Thus, this operation does not carry an increased risk of velopharyngeal insufficiency.

Controversy remains on the exact pathophysiology of OSA in children, but most specialists appear to accept the theory that upper airway patency is restricted or collapses during inspiration. Such collapse is the integrated result of a cluster of multifactorial interactions, which include functional and anatomical factors. In accordance with this theory, it is very important to attempt to maximally enlarge the narrowing and to decrease the collapsibility of the pharyngeal area by tightening the floppy airway, rather than removing only the adenoid and tonsillar tissues. Some authors ${ }^{23}$ have advocated the practice of suturing the tonsillar wound for a maximal enlargement of the retropalatal airspace, thereby decreasing the collapsibility of the lateral 
pharyngeal wall and soft palate. In this study, the long-term effects of suturing the tonsillar wound on relieving OSA remains to be observed.

\section{CONCLUSION}

In our study, tonsillectomy in conjunction with pharyngoplasty offers significant advantages in the postoperative period for pediatric patients with OSA. This procedure reduces the duration of wound healing and the ratio of postoperative hemorrhage, compared with tonsillectomy alone. Additionally, the risk of velopharyngeal insufficiency did not increase through this method. We consider that these results indicated that the combination of pharyngoplasty and tonsillectomy has great potential in the treatment of OSA in pediatric patients. Furthermore, its potential cost-saving advantage warrants continued study.

\section{REFERENCES}

1. Lumeng JC, Chervin RD. Epidemiology of pediatric obstructive sleep apnea. Proc Am Thorac Soc 2008; 5(2):24252.

2. Schwengel DA, Dalesio NM, Stierer TL. Pediatric obstructive sleep apnea. Anesthesiol Clin 2014;32(1):237-61.

3. Arali V, Namineni S, Sampath Ch. Pediatric obstructive sleep apnea syndrome: time to wake up. Int J Clin Pediatr Dent 2012; 5(1):54-60.

4. Zhu J, Fang Y, Chen X, et al. The impacts of obstructive sleep apnea hypopnea syndrome severity and surgery intervention on psychological and behavioral abnormalities and postoperative recovery in pediatric patients. Med Sci Monit 2014; 20:1474-80.

5. Slaats MA, Van Hoorenbeeck K, Van Eyck A, et al. Upper airway imaging in pediatric obstructive sleep apnea syndrome. Sleep Med Rev 2015; 21:59-71.

6. Mukhatiyar P, Nandalike K, Cohen HW, et al. Intracapsular and Extracapsular Tonsillectomy and Adenoidectomy in Pediatric Obstructive Sleep Apnea. JAMAOtolaryngol Head Neck Surg 2016; 142(1):25-31.

7. Reusser NM, Bender RW, Agrawal NA, et al. Posttonsillectomy hemorrhage rates in children compared by surgical technique. Ear Nose Throat J 2017; 96(7):e7-11.

8. Ferary M, Biet A, Strunski V, et al. Impact of the mode of hospitalisation on the postoperative complication rate after dissection tonsillectomy in children. Eur Ann Otorhinolaryngol Head Neck Dis 2014; 131(6):345-9.
9. Friedman M, Wilson MN, Friedman J, et al. Intracapsular coblation tonsillectomy and adenoidectomy for the treatment of pediatric obstructive sleep apnea/ hypopnea syndrome. Otolaryngol Head Neck Surg 2009; 140(3):358-62.

10. Kirazli T, Bilgen C, Midilli R, et al. Bipolar electrodissection tonsillectomy in children. Eur Arch Otorhinolaryngol 2005; 262(9):716-8.

11. D'Eredità R, Marsh RR. Contact diode laser tonsillectomy in children. Otolaryngol Head Neck Surg 2004; 131(5):732-5.

12. Shen $\mathrm{L}$, Zheng B, Lin Z, et al. Tailoring therapy to improve the treatment of children with obstructive sleep apnea according to grade of adenotonsillar hypertrophy. Int $J$ Pediatr Otorhinolaryngol 2015; 79(4):493-8.

13. Acar M, Kankilic ES, Koksal AO, et al. Method of the diagnosis of adenoid hypertrophy for physicians: adenoidnasopharynx ratio. J Craniofac Surg 2014; 25(5):e438-40.

14. Paulson LM, MacArthur CJ, Beaulieu KB, et al. Speech Outcomes after Tonsillectomy in Patients with Known Velopharyngeal Insufficiency. Int J Otolaryngol 2012; 2012:912767.

15. Tieu DD, Gerber ME, Milczuk HA, et al. Generation of consensus in the application of a rating scale to nasendoscopic assessment of velopharyngeal function. Arch Otolaryngol Head Neck Surg 2012; 138(10):923-8.

16. Amoils M, Chang KW, Saynina O, et al. Postoperative Complications in Pediatric Tonsillectomy and Adenoidectomy in Ambulatory vs Inpatient Settings. JAMA Otolaryngol Head Neck Surg 2016; 142(4):344-50.

17. Lou ZC, Lou ZH. Post-tonsillectomy hemorrhage: Underlying factors and prevention. Am J Otolaryngol 2018; 39(2):230-1.

18. Lehnerdt G, Senska K, Jahnke K, et al. Post-tonsillectomy haemorrhage: a retrospective comparison of abscessand elective tonsillectomy. Acta Otolaryngol 2005; 125(12):1312-7.

19. Mixon CM, Weinberger PM, Austin MB. Comparison of microdebrider subcapsular tonsillectomy to harmonic scalpel and eletrocautery total tonsillectomy. $A m \mathrm{~J}$ Otolaryngol 2007; 28(1):13-7.

20. Isaacson G. Tonsillectomy Care for the Pediatrician. Pediatrics 2012; 130(2):324-34.

21. Abdel-Aziz M. The effectiveness of tonsillectomy and partial adenoidectomy on obstructive sleep apnea in cleft palate patients. Laryngoscope 2012; 122(11):2563-7.

22. Mora R, Crippa B, Dellepiane M, et al. Effects of adenotonsillectomy on speech spectrum in children. Int $J$ Pediatr Otorhinolaryngol 2007; 71(8):1299-304.

23. Lim DJ, Kang SH, Kim BH, et al. Treatment of obstructive sleep apnea syndrome using radiofrequencyassisted uvulopalatoplasty with tonsillectomy. Eur Arch Otorhinolaryngol 2013; 270(2):585-93. 